\title{
Émile et la simplicité
}

\author{
Jérôme Brillaud ${ }^{1}$
}

Published online: 20 October 2016

(C) The Author(s) 2016. This article is published with open access at Springerlink.com

Rousseau, contrairement à Fénelon avec lequel il communie néanmoins en pensée, n'a jamais eu souci de définir la simplicité. Pourtant si le nombre d'occurrences d'un terme donne quelque mesure de son importance pour un auteur, force est d'admettre que la simplicité occupe une place centrale dans la pensée de Rousseau. À défaut de définition, le lecteur des œuvres de Jean-Jacques doit savoir se contenter de mentions de la simplicité où celle-ci est associée à un concept, ou une idée, qu'elle contribue à définir et qui, en retour, lui donne sens. Rousseau fait de la simplicité un concept opératoire mais ouvert, comblant ce creux sémantique dont il semble avoir saisi toute la profondeur par des notions connexes. Ainsi trouve-t-on des références à la vie simple, à la simplicité de mœurs ou encore à la simplicité naturelle, en somme à une simplicité toujours prédiquée à autre chose qu'ellemême. Au sujet de la simplicité André Comte-Sponville disait qu'on ne peut «l'approcher qu'indirectement, par autre chose qu'elle-même » (1995, p. 229). Tel est le cas chez Rousseau. Des premiers discours aux dernières œuvres autobiographiques, la simplicité est prédiquée, comme un prédicat qui en dit plus long sur le terme auquel on l'associe que sur lui-même, à la vertu, à la noblesse, à la liberté, à l'innocence, et à la nature.

Or si Rousseau se refuse à définir la simplicité, l'usage fréquent qu'il en fait est quant à lui parlant. La simplicité serait comme le non-dit de la pensée rousseauiènne. Non pas en ce qu'elle cacherait du sens mais bien en ce qu'elle dirait sans vraiment dire. Il s'agira dans cet article de voir comment la simplicité est instigatrice d'autres idées; comment par sa simple présence dans un énoncé elle donne naissance à autre chose qu'elle-même, au moins chez Rousseau. Pour ce faire, je me propose d'étudier la simplicité dans l'Émile car c'est dans ce traité, peut-être le plus achevé des textes de Rousseau, qu'elle revêt une forme à la fois

Jérôme Brillaud

jerome.brillaud@manchester.ac.uk

1 The University of Manchester, Oxford Road, Manchester M13 9PL, UK 
identifiable et originale. Originale en ce qu'elle se distingue de cette simplicité subordonnée à laquelle je faisais allusion et qui domine dans les autres œuvres de Rousseau. Identifiable pour la raison qu'elle n'est plus asservie à une notion connexe ou à l'un des nombreux topoï philosophiques de notre auteur. Dans l'Émile, on peut approcher la simplicité par elle-même. Pas de définition, certes, mais bel et bien un usage de la simplicité propre à Rousseau.

Pierre Burgelin est l'un des rares lecteurs de Rousseau à avoir consacré quelques pages à la simplicité. « D'une façon générale, écrit-il, la simplicité consiste essentiellement à refuser toute tyrannie de l'opinion » (1973, p. 275). Ce refus de l'opinion identifié par Burgelin ferait de l'homme simple un homme supérieur pour savoir se passer du jugement des autres et savoir toujours se suffire à lui-même. L'homme simple, tel qu'il est peint dans le Second Discours, est homme de bien et homme heureux. À Burgelin de rappeler que la simplicité est présente dès l'origine de l'œuvre de Rousseau, savoir la vision de Vincennes, vision qui avait révélé un âge d'or habité par des hommes « simples, sages, heureux », ceux-là même qui avaient en un instant réveillé l'espoir du philosophe dans le genre humain (Dialogues, pp. 828-829). On remarquera pourtant dans ce dernier exemple extrait des Dialogues où l'auteur relate avec force détails sa vision, que Rousseau ne propose aucun lien logique entre la simplicité, la sagesse et le bonheur comme si leur seule proximité dans la phrase suffisait à les faire entendre. «Simples, sages, heureux », qualificatifs qui sans être synonymes se complémentent ou plutôt se suppléent.

On distinguera trois principaux domaines de la pensée et de l'activité où la simplicité joue un rôle décisif: l'épistémologie, où il est question de pensée simple et méthode, chez Descartes notamment, la théologie et la divine simplicité qui occupe Fénelon entre autres et enfin l'esthétique et le style simple chez Boileau pour ne citer que lui. Cette dernière n'entrera pas dans mon propos car elle intéresse peu l'auteur de l'Émile. Un retour, certes bref, sur ces deux penseurs du simple que furent Descartes et Fénelon nous permettra de mieux apprécier ensuite toute l'originalité de l'emploi que fait Rousseau de la simplicité. ${ }^{1}$

La simplicité tient une place importante dans la pensée de Descartes. On lui a même reproché d'avoir engagé la philosophie sur une voie sans issue en fondant sa méthode précisément sur la simplicité. ${ }^{2}$ Pour Descartes, et sans entrer ici dans les détails, la simplicité est avant tout épistémologique, même s'il est vrai qu'il s'occupe à l'occasion de natures simples et de simplicité divine. ${ }^{3}$ Est simple, ce que l'on comprend aisément, ce qui se présente à l'esprit, le verbe « présenter » étant bien sûr ici lourd de sens. ${ }^{4}$ Dans son essai Le nouvel esprit scientifique Gaston

\footnotetext{
${ }^{1}$ Il y a peu d'histoires de la simplicité comme pratique philosophique et mode d'existence. Pour une présentation historique du concept, on pourra consulter le collectif Simple Living in History édité par S. Alexander et A. McLeod. Mais comme la plupart des ouvrages sur la simplicité, celui-ci omet Rousseau dont l'apport à la réflexion au sujet de la vie simple n'est pourtant pas négligeable, ne serait-ce que pour l'influence que son œuvre a exercée sur Thoreau et d'autres adeptes de la simplicité à partir du dixneuvième siècle.

${ }^{2}$ Voir notamment l'étude de Melany Mitchell, Complexity, a Guided Tour (Mitchell 2009a, p. ix).

${ }^{3}$ On consultera par exemple l'article de Dan Kaufman, « Divine Simplicity and the Eternal Truths in Descartes » (2003).

${ }^{4}$ Voir par exemple l'analyse détaillée de Stephen Menn (1998, p. 310-311).
} 
Bachelard, répondant à Descartes avec la verve et l'intelligence qu'on lui connait, ramassait sa critique du cartésianisme en une de ces formules choc essaimées dans son texte: «d'une manière générale, profère-t-il, le simple est toujours le simplifié » (1984, p. 143). Dans ce cas, surenchérit-il, il n’y aurait pas de simplicité en soi mais seulement des simplifications (1968, p. 99). Pour Bachelard, « la simplicité n'est pas un état des choses mais un véritable état d'âme. On ne croit pas parce que c'est simple, c'est simple parce que l'on croit » (1968, p. 101). Beaucoup d'historiens des sciences, sociologues et philosophes, lui donneront le la. Pour Edgar Morin, côté français, la simplicité entendue comme disjonction et réduction est doublement fautive. ${ }^{5}$ Côté américain, Mark Taylor et Sandra Mitchell font euxaussi campagne depuis quelques années contre l'esprit de simplicité. ${ }^{6}$ La simplicité, serait selon eux une affaire d'autrefois. Leur credo: nous vivons à l'heure de la complexité, et du changement et afin de comprendre la complexité qui nous entoure et à laquelle nous participons, il nous faut faire fi du passé et poser le scalpel de l'analyse sur le présent. Or ce que Mark Taylor n'a peut-être pas entièrement perçu c'est qu'en se penchant sur le présent pour apprécier toute la complexité de notre moment, ne faisons-nous pas la même erreur que ceux qui cherchaient à tout simplifier? Cette fois, nous compliquons les choses. Renversant la formule de Bachelard, on pourrait leur rétorquer que d'une manière générale, le complexe est toujours le complexifié. En somme, en matière de simplicité comme en matière de complexité, on trouve ce que l'on cherche, et seul le chercheur averti saura faire montre de prudence dans ses conclusions car, il le sait, sa méthode fonde son objet. Mais ce qui m'intéresse tout particulièrement dans cette trop brève histoire de la simplicité et du legs cartésien, c'est l'idée avancée par Bachelard que la simplicité est avant tout un état d'âme. C'est bien cet état d'âme qui prend corps dans les textes de Fénelon sur la simplicité et qu'à bien des égards on retrouvera plus tard chez Rousseau.

Fénelon et Rousseau se situent en marge de la réflexion épistémologique sur la simplicité. Elle est bien moins, pour eux, une méthode qu'une manière d'être au monde. Avant d'étudier le traité de pédagogie de Rousseau, quelques remarques liminaires sur la simplicité fénelonienne s'imposent. ${ }^{7}$ Car il me semble que la filiation, bien perçue d'ailleurs par de nombreux critiques, entre ces deux auteurs

\footnotetext{
5 « Ainsi, le paradigme de simplicité est un paradigme qui met de l'ordre dans l'univers, et en chasse le désordre. L'ordre se réduit à une loi, à un principe. La simplicité voit soit l'un, soit le multiple, mais ne peut voir que l'Un peut être en même temps le Multiple. Le principe de simplicité soit sépare ce qui est lié (disjonction), soit unifie ce qui est divers (réduction)» (2005, p. 79).

6 «We are living in a moment of unprecedented complexity, when things are changing faster than our ability to comprehend them. [...] To understand our time, we must comprehend complexity, and to comprehend complexity, we must understand what makes this moment different from every other. What distinguishes the moment of complexity is not change as such but rather the acceleration of the rate of change. [...] For many people, confusion and uncertainty create a desire for simplicity that leads to a futile longing to return to basic values and foundational beliefs. In today's world, however, simplicity has become an idle dream that no longer can be realized ». (Taylor 2001, p.3). Voir également l'essai de Sandra D. Mitchell, Unsimple Truths, Science, Complexity and Policy (2009b).

7 Au sujet de la simplicité dans l'œuvre de Fénelon, on consultera avec profit l'article de Jean-Louis Vieillard-Baron (1996) et le chapitre que lui consacre Vladimir Jankélévitch dans son Traité des vertus (1986, pp. 404-424).
} 
nous permettra de mieux comprendre la simplicité du philosophe genevois. ${ }^{8}$ Fénelon, qui selon d'Alembert et Chénier incarnait la simplicité, composa deux opuscules sur ce sujet. ${ }^{9}$ De la simplicité il dit qu'elle est « une droiture de l'âme qui retranche tout retour inutile sur elle-même et sur ses actions » (1983, p. 677). Il faut s'arrêter un instant sur cette définition. Pourquoi ce retour sur soi est-il inutile et contraire à la simplicité? Parce qu'à la question « qui suis-je? », Fénelon répondra: je suis un « assemblage de ce qui n'est plus, de ce qui cesse actuellement d'être, de ce qui n'est pas encore! C'est pourtant cette multitude de néants qui est ce que j'appelle le moi » (1997, p. 658). Une âme libre, précise Fénelon, qui «ne perd point son temps à raisonner sur ses pas, à les étudier, à regarder sans cesse ceux qu'elle a déjà faits, est la vraie simplicité » (1983, p. 678). Première remarque, pour l'archevêque de Cambrai, la simplicité se pense dans le rapport entre le moi et le présent. Or, comme le présent est un temps usurpé, « le présent même n'est pas à vous », Fénelon nous en avisait, du coup, le moi est lui aussi usurpé, tout relatif qu'il est (2007, p. 123). Ainsi à l'homme est-il interdit de prononcer un simple ego sum. «Qui suis-je? », taraudé par cette question, on l'aura compris, Fénelon répondra: « un je ne sais quoi qui finit dans l'instant même où il commence, en sorte que je ne puis jamais un seul moment me trouver moi-même fixe et présent à moimême pour dire simplement, je suis » (1997, pp. 663-664). Un remède, un seul: la simplicité comme exercice spirituel, ou «désappropriation », entendue comme un oubli de soi et un oubli du temps humain. En état de simplicité, comme l'a montré Georges Poulet, le présent, « cette petite éternité », l'expression est de Fénelon, se charge de présence divine (1968, p. 91). En état de simplicité donc, l'âme se dégrève de la pesanteur d'un passé lourd de sens et ne se laisse pas prendre au leurre d'un futur toujours incertain. Ce faiseur de conscience qu'était Fénelon a tôt fait de nous prévenir que « quiconque sort de cette simplicité [de cette simplicité divine qui investit l'âme] tombe de l'éternité dans le temps» (1997, p. 665). Mais la simplicité qui ouvre sur la « petite éternité » du présent risque à chaque instant de se verser dans l'histoire si l'on ne prend garde de vivre simplement, à savoir en totale désappropriation de soi et du temps.

En 1691, Fénelon donnait à Madame de Maintenon la directive suivante: «Allons selon que Dieu nous mène, au jour la journée » (Poulet, 1968, p. 94). Un autre directeur de conscience professera verbatim un demi-siècle plus tard: " le sage vit au jour la journée » (Solitaires, p. 911). Il s'agit de Jean-Jacques Rousseau qui plaçait cet aphorisme dans la bouche de l'Émile des Solitaires, texte inachevé mais ô combien riche d'enseignement si l'on peut se permettre le mot. Rousseau reconnaissait volontiers sa dette envers Fénelon, même s'il savait, le cas échéant, accuser la distance qui les séparait. La réflexion sur la simplicité et ses bienfaits dans l'éducation des enfants est amorcée très tôt dans l'Émile. Dès le premier livre, le gouverneur, pétri de nostalgie, se lamente sur le sort des enfants et l'éducation

\footnotetext{
${ }^{8}$ Voir entre autres études l'article de Marguerite Haillant à ce sujet (2001).

9 D'Alembert n'écrivait-il pas que «Fénelon a caractérisé lui-même en peu de mots cette simplicité qui le rendait si cher à tous les cœurs »? (1947, p. 767) En 1793, Marie-Joseph Chénier devait chanter les louanges de l'archevêque de Cambrai dans le discours préliminaire de la tragédie qu'il consacra au martyre du prélat. À l'heure de la révolution, grâce à « la simplicité de son âme » Fénelon mourait, non plus en délicatesse avec son roi, mais en odeur de sainteté révolutionnaire (2002, p. 247).
} 
qu'ils reçoivent. Il en arrive au constat suivant: « on ne sait plus être simple en rien; pas même autour des enfants » (Émile, p. 292). Le luxe dont on les comble et les atours dont on les affuble sont nocifs à leur développement et à leur santé car, de l'avis du gouverneur, seul « l'homme vivant dans la simplicité primitive est sujet à peu de maux » (Émile, p. 588). Antienne primitiviste qu'il entonnera bien des fois. À la fin du roman ne lit-on pas que « l'étude des divers peuples dans leurs provinces reculées, et dans la simplicité de leur génie originel »montre que plus ces nations «se rapprochent de la nature, plus la bonté domine dans leur caractère » (Émile, p. 852). La simplicité naturelle, garante de la santé du corps et de l'esprit, s'inscrit au cœur du projet pédagogique. Il en rappelait volontiers les bienfaits à son élève: « en t'élevant dans la simplicité de la nature, au lieu de te prêcher de pénibles devoirs, je t'ai garanti des vices qui rendent ces devoirs pénibles » (Émile, p. 818). Le respect de la simplicité de la nature comme premier principe pédagogique fait toute l'originalité de l'éducation d'Émile et d'un ambitieux projet d'éducation dont le roman pose les assises.

L'association entre nature et simplicité traverse la pensée pédagogique de Rousseau. Or pour en comprendre les enjeux et les fins, il faut s'arrêter sur le premier terme de la relation: la nature. «Uniquement dans la philosophie de Rousseau, écrit Paul Audi, le terme communément employé de « nature » désigne la dimension d'origine de toute manifestation possible. Autant dire que par son concept ce nom se réfère à un Commencement qui, lui, n'est pas à entendre historiquement [...] mais de façon authentiquement phénoménologique, comme un certain type de «présence »»(2008, pp. 18-19). C'est cette manifestation première, ce surgissement, qui pose les conditions de l'avènement de l'être. Dans la métaphysique traditionnelle, «être, rappelle Paul Audi, ne veut pas dire autre chose que: «être présent», ou «être présent à soi »(2008, p. 28). Mais il convient de préciser que Rousseau n'a que faire de cette « présence à soi » lorsque celle-ci désigne un rapport, donc une duplicité, entre le moi présent [étymologiquement «devant moi »] et le moi-même. Ce que recherche Rousseau, c'est une «présence absolue », non relative et intransitive, une présence où le moi et le moi-même ne formeraient plus qu'un. Se pose alors la question des conditions de la symbiose du moi et du moi-même, de cette présence absolue, de cette "intériorité absolue » (Audi, 2008, p. 19). Il revient à Émile des Solitaires d'apporter un premier élément de réponse à cette question.

Trahi par Sophie et ne se berçant plus de douces illusions sur le compte de celle qu'il aimait, Émile recouvre le bonheur en s'abandonnant au présent: «j'avais fait un grand pas vers le repos. Délivré de l'inquiétude de l'espérance, et sûr de perdre ainsi peu à peu celle du devoir, en voyant que le passé ne m'était plus rien, je tâchais de me mettre tout à fait dans l'état d'un homme qui commence à vivre. Je me disais qu'en effet nous ne faisions jamais que commencer, et qu'il n'y a point d'autre liaison dans notre existence qu'une succession de moments présents, dont le premier est toujours celui qui est en acte. Nous mourrons et nous naissons chaque instant de notre vie » (Solitaires, p. 905). Rousseau répond comme en écho à Fénelon en gageant le repos de l'âme sur l'abandon total dans le présent, présent entendu comme sentiment de l'acte en cours, comme naissance permanente. Mais ce n'est pas tout. L'abandon dans le présent est hypothéqué sur un désaveu du passé: «j’ai 
bu l'eau d'oubli; le passé s'efface de ma mémoire et l'univers s'ouvre devant moi », disait Émile (Solitaires, p. 912). Chaque renaissance, autrement dit chaque instant, est aussi un oubli. ${ }^{10}$

Si ce sentiment du présent peut soulager les tourments de l'âme inquiète, celle-ci ne peut être entièrement apaisée que lorsque le corps est lui aussi au repos. Ce «pas vers le repos » qu'Émile venait de faire est à prendre de manière littérale. "Que fais-je? où vais-je? quel est mon but? »se demande-t-il (Solitaires, p. 914). À l'homme trahi de trouver et sérénité et réconfort dans le repos physique: « je fais ma tâche, je reste à ma place, j'use avec innocence et simplicité cette courte vie » (Solitaires, p. 914). Si la simplicité est repos dans le moment présent, elle rejette en même temps la futilité de la course. De cette éducation basée sur la simplicité des gestes et des pensées, l'élève avait retenu une chose: « un des fruits de mon éducation était d'être pris au mot sur ce que je me donnais pour être, et rien de plus, parce que j'étais simple en toute chose, et qu'en remplissant un poste je n'en briguais pas un autre. Ainsi, j'étais toujours à ma place » (Solitaires, p. 913). Il s'agit moins ici de savoir sa place et de s'y tenir, que de pouvoir être pleinement en place. Tout en manifestant sa gratitude envers son gouverneur, il s'expliquait en ces mots: « je n'ai rien tant appris de vous dès mon enfance qu'à être toujours tout entier où je suis, à ne jamais faire une chose et rêver à une autre » (Solitaires, p. 899). Être simple consiste donc à savoir sa place et y demeurer de façon à être tout entier non pas à soi, non pas avec soi, mais en soi. «Pourvu non avec moi, mais en moi, dit Émile, de plus de ressources que je n'avais besoin pour vivre, je n'étais embarrassé ni de voiture, ni de ma subsistance »(Solitaires, p. 913). Ainsi, être simple signifie être présent en soi, ici et maintenant.

Il faudrait se garder de lire ici un quelconque appel à la quiétude ou à un quelconque retrait du monde. Chez Rousseau, et plus particulièrement dans l'Émile, la simplicité comme présence en soi demeure tributaire de l'activité, du métier, et du geste qui déterminent le présent comme lieu et moment. Car l'une des propriétés de ce type de présence absolue est d'être corporelle. L'analyse de Hans Ulrich Gumbrecht en rend compte. Au sujet de cette présence qu'il qualifie d' " extrême temporalité », Gumbrecht note: «ce qui est «présent » pour nous (notion très proche du latin prae-esse) est devant nous, à portée de main et de toucher » (2010, p. 38).

On sait que l'éducation d'Émile porte en grande partie sur l'éveil puis la formation des sens, notamment le toucher. Pour Rousseau, le toucher est le sens le plus sûr, ou le moins fautif, parce qu'il s'étend moins loin que la vue par exemple: « les jugements du tact, explique-t-il, sont les plus surs [...] ils rectifient l'étourderie des autres sens, qui s'élancent au loin sur des objets qu'ils aperçoivent à peine, au lieu que tout ce qu'aperçoit le toucher, il l'aperçoit » (Émile, p. 389). Parce qu'il se limite au périmètre immédiat de la personne, le toucher est non seulement le plus fiable des sens, il est également celui « qui nous instruit le mieux de l'impression que les corps étrangers peuvent faire sur le nôtre [...] et nous donne le plus immédiatement la connaissance nécessaire à notre conservation » (Émile,

\footnotetext{
${ }^{10}$ Deux belles études abordent la question du moment et du présent chez Rousseau: Thomas M. Kavanagh, Esthetics of the Moment et Laurence Mall, Émile ou les figures de la fiction.
} 
p. 389). Ce sont les mots du gouverneur. Sens fiable car restreint dans sa portée et dans son activité, le toucher est également le sens le plus important pour la conservation de soi.

Selon la hiérarchie des sens établie par le gouverneur d'Émile, le toucher s'oppose à la vue comme le simple s'oppose au composé. En effet, à force d'exercice, à force de simplification sensorielle, la sensation tactile peut se passer du concours des autres sens pour rendre un objet fidèlement. En revanche la vue reste irrémédiablement tributaire des autres sens. Pour former la vue, nous apprend le gouverneur, « il faut donc suivre une méthode contraire à la précédente; au lieu de simplifier la sensation, la doubler, la vérifier toujours par une autre, assujettir l'organe visüel à l'organe tactile, et réprimer, pour ainsi dire, l'impétuosité du premier sens par la marche pesante et réglée du second »(Émile, p. 392). Simplifier la sensation, c'est-à-dire la limiter à l'usage d'un seul sens, et ainsi éviter l'encombrement des autres sens qui perturberaient la perception. En bref, sens simple car sens autonome, le toucher est le plus juste et le plus sûr des sens.

À cette sensation simple, car univoque, qu'est le toucher viennent s'ajouter les idées simples, définies comme suit: « les idées simples ne sont que des sensations comparées. Il y a des jugemens dans les simples sensations aussi bien que dans les sensations complexes [la vue par exemple], que j'appelle idées simples » (Émile, p. 481). L'idée simple se distingue de la sensation simple par la nature du jugement opéré: le jugement sensoriel est passif (je sens ce que je sens), alors que le jugement intellectuel est actif. Il agit par comparaisons, rapprochements, et rapports. Mais l'enseignement d'Émile ne se s'arrête pas à cette taxonomie. « La meilleure maniére d'apprendre à bien juger, poursuit-il, est celle qui tend le plus à simplifier nos expériences et à pouvoir même nous en passer sans tomber dans l'erreur. D'où il suit qu'après avoir longtems vérifié les rapports des sens l'un par l'autre, il faut encore apprendre à vérifier les rapports de chaque sens par lui-même, sans avoir besoin de recourir à un autre sens. Alors chaque sensation deviendra pour nous une idée, et cette idée sera toujours conforme à la vérité » (Émile, p. 484). L'étude des rapports externes, les sens entre eux, doit conduire à l'étude des rapports internes de chaque sens. Une éducation réussie, qui aura simplifié les expériences, débouchera sur la conclusion suivante: la sensation et l'idée ne font plus qu'un, le « je sens ce que je sens » équivaut au « je pense ce que je pense » dans l'expérience simplifiée. Ultime étape, on se passera de l'expérience pédagogique, heuristique, pour arriver à cette fusion de l'idée et de la sensation. L'esprit ainsi entraîné reçoit désormais le monde directement, non pas passivement, mais immédiatement dans le double acte intellectuel et sensoriel.

Cet être simple que Rousseau campe dans l'Émile ressemble en bien des traits à l'ivrogne, et à l'idiot, dont Clément Rosset a brossé le portrait dans son essai sur le réel: « l'ivrogne perçoit simple, et c'est plutôt l'homme sobre qui, habituellement, perçoit double. L'ivrogne est, quant à lui, hébété par la présence sous ses yeux d'une chose singulière et unique qu'il montre de l'index tout en prenant l'entourage à témoin, et bientôt à partie si celui-ci se rebiffe: regardez là, il y a une fleur, c'est une fleur, c'est une fleur, mais puisque je vous dis que c'est une fleur...Une chose toute simple, c'est-à-dire saisie comme singularité stupéfiante, comme émergence insolite dans le champ de l'existence » (1997, p. 41). Seul l'être simple est capable de saisir 
cette émergence de la chose dans toute sa singularité. Ce fut le cas pour Rousseau à Vincennes à en croire le rapport qu'il fait de son expérience.

Ainsi, la simplicité dans l'Émile prend plusieurs formes. Elle consiste en premier lieu en une simplification ou un resserrement de l'être tout entier présent en soi, oublieux de son histoire, passée et future. En second lieu, la simplicité résulte de l'union des perceptions sensorielles et intellectuelles, union obtenue par une simplification de l'activité des sens et du jugement, notamment du toucher. Dans un cas comme dans l'autre, on pourrait parler de contraction autour d'un point absolument central, et unique, le moi. Comme l'indiquait Émile, ce moi simple car un - est en état de perpétuelle naissance: " nous mourrons et nous naissons chaque instant de notre vie » (Solitaires, p. 905). Jean-Luc Nancy appelait cet état « présence ». Pour Nancy, comme l'a bien vu Gumbrecht, la présence est une naissance, une apparition/disparition simultanée. ${ }^{11}$ L'être simple tel que le peint Rousseau, n'est pas un être en devenir — devenir pointe trop vers une finalité mais un être qui est présent, autrement dit, un être toujours naissant.

Open Access This article is distributed under the terms of the Creative Commons Attribution 4.0 International License (http://creativecommons.org/licenses/by/4.0/), which permits unrestricted use, distribution, and reproduction in any medium, provided you give appropriate credit to the original author(s) and the source, provide a link to the Creative Commons license, and indicate if changes were made.

\section{Références}

Alexander, S. et McLeod, A. (Éds. Gén.). (2014). Simple living in history. Melbourne: The Simplicity Institute.

Audi, P. (2008). Rousseau: une philosophie de l'âme. Paris: Verdier.

Bachelard, G. (1968). Essai sur la connaissance approchée. Paris: Vrin.

Bachelard, G. (1984). Le nouvel esprit scientifique. Paris: Presses universitaires de France. Quadrige.

Burgelin, P. (1973). La philosophie de l'existence de J.-J. Rousseau. Paris: Vrin.

Chénier, M.-J. (2002). Fénelon. In G. Ambrus \& F. Jacob (Éds.), Théâtre. Paris: Flammarion.

Comte-Sponville, A. (1995). Petit traité des grandes vertus. Paris: Presses Universitaires de France.

D’Alembert, J. 1. R. (1947). Éloge de Fénelon. Paris: Bordas.

Fénelon, F. de Salignac de La Mothe-Fénelon, dit Fénelon. (1983). In Jacques Le Brun (Éd.), Euvres. (vol. I), Paris: Gallimard. Pléiade.

Fénelon, F. de Salignac de La Mothe-Fénelon, dit Fénelon. (1997). In Jacques Le Brun (Éd.), Euvres. (vol. II), Paris: Gallimard. Pléiade.

Fénelon, F. Salignac de La Mothe-Fénelon, dit Fénelon. (2007). Lettres spirituelles. In Le Brun, J., Neveu, B. et Noye, I. (Éds.), Correspondance de Fénelon. (vol. XVIII), Genève: Droz.

Gumbrecht, H. U. (2010). Éloge de la présence. Paris: Libella Maren Sell.

Haillant, M. (2001). Rousseau admirateur de Fénelon: Ressemblances et différences. In R. Thiéry (Éd.), Jean-Jacques Rousseau politique et nation. Paris: Champion.

Jankélévitch, V. (1986). Traité des vertus III. L’innocence et la méchanceté. Paris: Flammarion. Champs essais.

Kaufman, D. (2003). Divine simplicity and the eternal truths in Descartes. British Journal of the History of Philosophy, 11(4), 553-579.

Kavanagh, T. M. (1996). Esthetics of the moment. Literature and art in the French Enlightenment. Philadelphia: University of Pennsylvania Press.

Mall, L. (2002). Émile ou les figures de la fiction. Oxford: Voltaire Foundation.

\footnotetext{
11 « Presence itself is birth, the coming that effaces itself and brings itself back » . (1993, p. 5) Voir l'analyse de Gumbrecht (2010, p. 96).
} 
Menn, S. (1998). Descartes and Augustine. Cambridge: Cambridge University Press.

Mitchell, M. (2009a). Complexity, a guided tour. Oxford: Oxford University Press.

Mitchell, S. D. (2009b). Unsimple truths, science, complexity and policy. Chicago: University of Chicago. Morin, E. (2005). Introduction à la pensée complexe. Paris: Seuil. Points-Essais.

Nancy, J.-L. (1993). The birth of presence. Stanford: Stanford University Press.

Poulet, G. (1968). La mesure de l'instant. Paris: Plon.

Rosset, C. (1997). Le réel, traité de l'idiotie. Paris: Minuit.

Rousseau, J. J. (1959). Rousseau juge de Jean Jaques, Dialogues. In B. Gagnebin, M. Raymond, \& R. Osmont (Éds.), Euvres complètes (Vol. I). Paris: Gallimard. Pléiade.

Rousseau, J. J. (1969a). Émile. In B. Gagnebin \& M. Raymond (Éds.), Euvres complètes (Vol. IV). Paris: Gallimard. Pléiade.

Rousseau, J. J. (1969b). Émile et Sophie ou les solitaires. In B. Gagnebin \& M. Raymond (Éds.), Euvres complètes (Vol. IV). Paris: Gallimard. Pléiade.

Taylor, M. C. (2001). The moment of complexity. Chicago: The University of Chicago Press.

Vieillard-Baron, J. L. (1996). De la simplicité. In D. Leduc-Fayette (Éd.), Fénelon, philosophie et spiritualité (pp. 209-224). Droz: Genève. 